\title{
EFICIENCIA EN EL USO DE LA RADIACIÓN POR HÍBRIDOS DE MAÍZ DE VALLES ALTOS DE MÉXICO
}

\author{
RADIATION USE EFFICIENCY BY MAIZE HYBRIDS OF THE MEXICAN HIGH VALLEYS
}

\author{
Alejandra Contreras Rendón, Carlos G. Martínez Rueda* y Gaspar Estrada Campuzano
}

Facultad de Ciencias Agrícolas, Universidad Autónoma del Estado de México, Campus Universitario El Cerrillo. 50200 Toluca, México. Tel. (Fax): (722) 29655-18. Ext. 142.

*Autor para correspondencia (cgmartinezr@uaemex.mx)

\section{RESUMEN}

En maíz (Zea mays L.), la intercepción y utilización de la radiación solar a nivel de cultivo son dos procesos que influyen en la producción de biomasa y el rendimiento de grano. El objetivo de esta investigación fue estudiar el impacto de la fecha de siembra, la densidad de población y la disponibilidad de nitrógeno, sobre la radiación interceptada (RI), eficiencia en el uso de la radiación (EUR) y el rendimiento de grano en tres híbridos de maíz para los Valles Altos de México. Los experimentos se llevaron a cabo en el ciclo primavera verano 2008 en Toluca, México. Se evaluaron tres híbridos de maíz ('H48', 'H50' y 'Cóndor'), en dos fechas de siembra (intermedia, 11/04/08; y tardía 30/04/08), bajo dos niveles de fertilización nitrogenada $\left(30\right.$ y $\left.180 \mathrm{~kg} \mathrm{~N} \mathrm{ha}^{-1}\right)$ y dos densidades de población (6.25 y 9.25plantas $\left.\mathrm{m}^{-2}\right)$. Para cada combinación de fecha de siembra y dosis de $\mathrm{N}$ se estableció un experimento bajo un diseño de parcelas divididas con tres repeticiones, donde se asignó a la densidad de población a las parcelas grandes y los híbridos a las parcelas chicas. Los resultados obtenidos indican que el manejo agronómico y el potencial genético de los híbridos influyeron significativamente sobre la eficiencia en el uso de la radiación y el rendimiento de grano del maíz. Cuando se aumentó la disponibilidad de $\mathrm{N}$ y la densidad de población el cultivo interceptó mayor cantidad de radiación solar $(\mathrm{P} \leq$ 0.05), con lo que se obtuvo un mayor número de granos por unidad de superficie. El rendimiento de grano de los tres híbridos fue explicado por el número de granos por unidad de superficie, más que por el peso individual de los granos. El híbrido 'Cóndor' fue el genotipo que produjo el mayor número de granos.

Palabras clave: Zea mays, biomasa, número de granos, radiación interceptada, rendimiento de grano.

\section{SUMMARY}

The interception and use of solar radiation by maize (Zea mays L.) at canopy level, are two processes that directly influence the production of biomass and grain yield. The aim of this work was to study the effect of planting date, population density and nitrogen doses on intercepted radiation (IR), radiation use efficiency (RUE) and grain yield of highlands maize hybrids. The experiments were carried out in the 2008 Spring-Summer season in Toluca, México. Three maize hybrids ('H48', 'H50' and 'Condor'), two sowing dates, (intermediate $(4 / 11 / 08)$ and late $(30 / 04 / 08))$, two nitrogen fertilization levels ( 30 and $180 \mathrm{~kg} \mathrm{~N} \mathrm{ha}^{-1}$ ) and two population densities (6.25 and 9.25 plant $\mathrm{m}^{-2}$ ) were evaluated. For each combination of planting date and $\mathrm{N}$ level, an experiment was carried out under a split plot design with three replications, assigning the population density to main plots and hybrids to subplots. Results indicated that agronomic management practices and the genetic potential of hybrids significantly influenced $(P \leq 0.05)$ on radiation use efficiency and grain yield of these highland maize hybrids. When $\mathrm{N}$ doses and population density increased the intercepted radiation by the plant canopy also increased, and this enhanced the growth conditions for setting a larger number of grains per unit area. Grain yield of the three hybrids was explained by number of grains per unit area rather than by average grain weight. The hybrid 'Condor' produced a larger number grain per area unit.

Index words: Zea mays, biomass, grain number, grain yield, intercepted radiation.

\section{INTRODUCCIÓN}

La intercepción y la utilización de la radiación solar son dos procesos fundamentales que rigen el crecimiento y rendimiento de los cultivos (Monteith, 1977). En maíz (Zea mays L.), el rendimiento de grano (RG) puede estimarse mediante el producto de la materia seca (MS) acumulada hasta la madurez fisiológica por el índice de cosecha. La MS a su vez es el resultado de la intercepción y utilización de la radiación solar incidente sobre el dosel del cultivo durante su ciclo ontogénico (Andrade y Ferreiro, 1996; Lee y Tollenaar, 2007). La radiación solar fotosintéticamente activa (RFA) interceptada por los cultivos es transformada en biomasa, de forma que la pendiente de la relación lineal entre ambas variables 
representa la eficiencia en el uso de la radiación (EUR), normalmente expresada en $\mathrm{g}$ de $\mathrm{MS} \mathrm{MJ}^{-1}$ interceptado (Sinclair y Muchow, 1999). En diversos estudios en maíz la EUR del maíz varía de 2 a $3.4 \mathrm{~g} \mathrm{MJ}^{-1}$ variación que depende del híbrido, de la disponibilidad de $\mathrm{N}$ y del ambiente de producción (Birch et al., 1999).

Las prácticas de manejo agronómico tienen un papel importante en la determinación del rendimiento final en el cultivo de maíz, como lo es la fecha de siembra, porque puede modificar sensiblemente la duración del ciclo ontogénico y la capacidad de captura de radiación solar; esto impacta la producción de MS y el RG (Otegui et al., 2003). La densidad de población también impacta en el desarrollo vegetativo y reproductivo del maíz, que a su vez influyen directamente en el uso de los recursos, que incluye los niveles de captura y utilización de la radiación solar, agua y nutrimentos (Cárcova et al., 2003). Otro factor importante para determinar RG es la disponibilidad de $\mathrm{N}$, cuyo aporte en condiciones de deficiencia mejora el crecimiento foliar y aumenta la cantidad de radiación interceptada por el cultivo, lo que aumenta la tasa de crecimiento en el periodo cercano a la floración (D'Andrea et al., 2008).

Es conveniente entonces estudiar la respuesta de los atributos ecofisiológicos que determinan el rendimiento de grano ante las variaciones ambientales, para identificar las mejores prácticas de manejo y optimizar el uso de los recursos en una localidad o región (Andrade et al., 2005; Edwards et al., 2005). En el Valle de Toluca, México se dispone de escasa información sobre los atributos ecofisiológicos que determinan el RG en respuesta a factores ambientales y de manejo agronómico. El objetivo del presente trabajo fue estudiar el impacto de la fecha de siembra, la densidad de población y la disponibilidad de nitrógeno, sobre la cantidad de radiación interceptada, la eficiencia en el uso de la radiación (EUR) y el rendimiento de grano en híbridos de maíz para Valles Altos.

\section{MATERIALES Y MÉTODOS}

\section{Sitio experimental}

Los experimentos se establecieron durante el ciclo agrícola primavera-verano 2008, tanto con riego a la siembra como en secano, en la localidad de "El Cerrillo Piedras Blancas", Toluca, México (99 41' 30" LO y 19²4'
34" LN), a una altitud de $2607 \mathrm{~m}$. El clima de esta localidad es $\mathrm{C}(\mathrm{w} 2)(\mathrm{w}) \mathrm{b}(\mathrm{i})$, que de acuerdo con García (1988) corresponde al tipo templado subhúmedo con lluvias en verano y poca oscilación térmica. El suelo predominante es de tipo vertisol y se caracteriza por tener una textura franco-arcillosa. El terreno donde se establecieron los experimentos no fue cultivado en el ciclo anterior; el perfil de suelo de 0 a $60 \mathrm{~cm}$ presentó $0.17 \%$ de nitrógeno inicial (equivalente a $162 \mathrm{~kg} \mathrm{~N} \mathrm{ha}^{-1}$ ) y $1.0 \%$ de materia orgánica.

\section{Factores de estudio y diseño experimental}

Se evaluaron tres híbridos comerciales de maíz de cruza trilineal ('H48', 'H50' y 'Cóndor') en dos fechas de siembra (intermedia: 11/04/08, y tardía: 30/04/08), dos niveles de fertilización nitrogenada $\left(\mathrm{N}_{1}: 30\right.$ y $\mathrm{N}_{2}: 180 \mathrm{~kg} \mathrm{~N}$ $\left.\mathrm{ha}^{-1}\right)$, y dos densidades de población $\left(\mathrm{D}_{1}: 62500\right.$ y $\mathrm{D}_{2}: 92$ 500 plantas $\mathrm{ha}^{-1}$ ). Para cada combinación de fecha de siembra y nivel de $\mathrm{N}$ se estableció un diseño de parcelas divididas con tres repeticiones, en el que las densidades de población se asignaron a las parcelas grandes y los híbridos a las parcelas chicas, que estuvieron conformadas por cuatro surcos de $6 \mathrm{~m}$ de longitud y $0.80 \mathrm{~m}$ de distancia entre surcos. La parcela experimental útil estuvo constituida por los $4 \mathrm{~m}$ interiores de los dos surcos centrales.

\section{Condiciones generales del experimento}

En la siembra del 11/04/2008 se aplicó un riego por goteo para favorecer la germinación de la semilla, mientras que la segunda fecha de siembra (30/04/2008) se hizo en condiciones de temporal (secano) durante todo el ciclo de cultivo. En la etapa V4 (cuatro hojas expandidas) se marcaron cinco plantas en la parte central de las parcelas, y se registró su fenología de acuerdo con la escala propuesta por Ritchie y Hanway (1982). Las fechas de emergencia, antesis (por lo menos una antera visible) y floración femenina (por lo menos un estigma visible) se registraron en todas las plantas marcadas; la madurez fisiológica se registró cuando el grano alcanzó su peso máximo (R8). Se utilizaron como fuentes de N, P y K a los siguientes fertilizantes: urea $(46 \% \mathrm{~N})$, superfosfato de calcio triple $\left(46 \% \mathrm{P}_{2} \mathrm{O}_{5}\right)$ y cloruro de potasio $\left(60 \% \mathrm{~K}_{2} \mathrm{O}\right)$ respectivamente; a la siembra se aplicó la dosis de $30 \mathrm{~N}$ 60P-30K para el nivel bajo de fertilización nitrogenada y la dosis de $60 \mathrm{~N}-60 \mathrm{P}-30 \mathrm{~K}$ para el nivel alto de fertilización. 
Luego, en las etapas V6 y V10 se aplicaron $60 \mathrm{~kg}$ de $\mathrm{N} \mathrm{ha}^{-1}$ en los tratamientos con nivel alto de fertilización con $\mathrm{N}$ (180 kg de $\left.\mathrm{N} \mathrm{ha}^{-1}\right)$. Las malezas fueron controladas con una aplicación de Gesaprim Calibre $90 \AA$ (i.a. atrazina) a dosis de $2 \mathrm{~L} \mathrm{ha}^{-1}$, más deshierbes manuales. No se presentaron ataques significativos de plagas $o$ enfermedades durante el ciclo del cultivo. Durante la estación de crecimiento se registró temperatura, precipitación y radiación solar mediante una estación climática (DAVIS Instrument Co., USA) ubicada a $200 \mathrm{~m}$ del sitio experimental.

\section{Variables medidas}

La acumulación de materia seca (MS) se determinó mediante muestreos destructivos; las estructuras de las plantas fueron secadas en una estufa a $70{ }^{\circ} \mathrm{C}$ por $72 \mathrm{~h}$, hasta alcanzar peso constante. En las etapas V4, V6, V8, V12 y R1 de la escala de Ritchie y Hanway (1982) se cosechó una planta con competencia completa por parcela y repetición. En la madurez fisiológica (R8) se cosecharon diez plantas con competencia completa en cada parcela experimental útil.

Los datos de radiación solar incidente se obtuvieron de la estación meteorológica y fueron multiplicados por el factor 0.48 para transformarlos a radiación fotosintéticamente activa $\left(\mathrm{MJ} \mathrm{m}^{-2}\right)$, y se expresaron en $\mathrm{MJ} \mathrm{m}^{-2} \mathrm{~d}^{-1}$ (Birch et al., 1999). El porcentaje de radiación fotosintéticamente activa interceptada (RFA) se midió periódicamente (tres veces por semana) a partir de la etapa V4, de acuerdo con Gallo y Daughtry (1986), con un sensor lineal cuántico (Ceptómetro BAR-RAD DUAL USB , Cavadevices ${ }^{\circledast}$, Bs.As. Argentina); se tomaron cinco lecturas por parcela entre las 11:30 y las 14:30 h en días despejados. Para esto el sensor se colocó diagonalmente entre dos entre surcos sobre el dosel para obtener la radiación incidente (Io) y debajo del dosel para medir la radiación transmitida (It). El porcentaje de radiación interceptada $(R I)$ se calculó con la siguiente ecuación:

$$
R I=[(I o-I t) / I o] \times 100
$$

El porcentaje de intercepción diario se obtuvo mediante la interpolación lineal entre dos mediciones; después la RFA incidente por día se multiplicó por el porcentaje de intercepción diario correspondiente, para obtener la RFA interceptada diaria la cual fue acumulada de emergencia a floración, de floración a madurez fisiológica y de emergencia a madurez fisiológica. Para los periodos de emergencia a madurez fisiológica y de emergencia a floración, la eficiencia en el uso de la radiación (EUR, $\mathrm{g} \mathrm{M}^{-1}$ ) se calculó mediante modelos de regresión lineal simple con intercepto forzado al origen $[Y$ $=b(X)]$, mientras que para el periodo de floración a madurez, la EUR se calculó a partir de modelos de regresión lineal simple sin forzar el intercepto al origen $[Y$ $=a+b(X)]$. En los dos casos anteriores la EUR estuvo representada por la pendiente (b) de la relación lineal entre la MS (Y) y la radiación solar fotosintéticamente activa interceptada por parcela (X).

\section{Rendimiento de grano y componentes del rendimiento}

El número de granos por planta (NGP) se calculó con un contador electrónico de granos Seedburo ${ }^{\circledast} 801$ Count A Pack, (IL,USA). Previo a medir el peso de grano por planta (PGP) las muestras fueron secadas en una estufa a $70{ }^{\circ} \mathrm{C}$ hasta alcanzar peso constante, sin considerar las mazorcas enfermas). El peso de mil granos (PMG) se calculó al dividir el PG sobre el NGP y luego se multiplicó por 1000. El número de granos por $\mathrm{m}^{2}(\mathrm{NG})$ se obtuvo de sumar el NGP y dividirlo entre la superficie que abarcaron las plantas. El rendimiento de grano por parcela (RG) se ajustó a $14 \%$ de humedad en el grano.

\section{Análisis estadístico}

Con la información obtenida de las variables de estudio, se hicieron los análisis de varianza individuales para cada experimento (fecha de siembra y dosis de nitrógeno), bajo el modelo estadístico del diseño experimental de parcelas divididas. También se hizo el análisis combinado de los cuatro experimentos para estudiar los efectos principales (fecha de siembra, dosis de nitrógeno, genotipo y densidad de población), así como las interacciones de primero, segundo y tercer orden.

Para describir la relación entre la radiación interceptada acumulada durante el periodo crítico (RIAPC), que para el caso del maíz ocurre durante los 15 $\mathrm{d}$ antes y $15 \mathrm{~d}$ después de la floración femenina (Andrade et al., 2005) con el número de grano por $\mathrm{m}^{2}$ (NG), se utilizó un modelo de regresión bilineal con fase estacionaria, cuyos parámetros se estimaron de la siguiente forma: 


$$
\begin{aligned}
& N G=a+b x \text { para } x<c, \\
& N G=a+b c \text { para } x \geq c .
\end{aligned}
$$

Donde: $\mathrm{NG}=$ Número de granos por $\mathrm{m}^{2} ; x=$ Radiación interceptada acumulada en el periodo crítico $\left(\mathrm{MJ} \mathrm{m}^{-2}\right) ; a$ = intercepto; $b=$ pendiente; $c=$ Radiación interceptada acumulada en el periodo crítico para el cual se alcanza la fase estacionaria.

El análisis estadístico de los datos, se hizo con el programa SAS ver. 8.12 (SAS Institute, 1999) y el programa TBLCURVE Versión 2.0 (Jandel AISN Software, 1992).

\section{RESULTADOS Y DISCUSIÓN}

\section{Condiciones climáticas}

Durante la estación de crecimiento de las dos fechas de siembra, las condiciones climáticas estuvieron caracterizadas por una precipitación total inusualmente alta $(1122 \mathrm{~mm})$; la lluvia se distribuyó uniformemente durante la mayor parte del ciclo de cultivo, con una temperatura promedio de $13.3{ }^{\circ} \mathrm{C}$ y una radiación solar incidente promedio de $19 \mathrm{MJ} \mathrm{m}^{-2} \mathrm{~d}^{-1}$ (Cuadro 1). En el mes de noviembre ocurrieron temperaturas por debajo de $0 \quad{ }^{\circ} \mathrm{C}$, cuando el cultivo había alcanzado la etapa de madurez fisiológica, lo que permite afirmar que durante los experimentos no se presentaron condiciones de estrés hídrico o bajas temperaturas que pudieran haber interactuado con los tratamientos del presente trabajo.

\section{Radiación interceptada y eficiencia en el uso de la radiación}

En la fecha de siembra tardía disminuyó en 32\% ( $\mathrm{P} \leq$ 0.05 ) la cantidad de radiación interceptada (RI) en el periodo previo a floración (EM-FF), pero se incrementó en $29 \%(\mathrm{P} \leq 0.01)$ durante el periodo postfloración (FF$\mathrm{MF})$. Sin embargo, no se observaron diferencias significativas $(\mathrm{P}>0.05)$ entre las dos fechas de siembra en la cantidad de RI durante todo el ciclo de cultivo (EMMF). Cuando se incrementó la dosis de $\mathrm{N}$ de 30 a $180 \mathrm{~kg}$ ha $^{-1}$ la RI aumentó en $19 \%(\mathrm{P} \leq 0.05)$ durante el periodo FF-MF y en $12 \%(\mathrm{P} \leq 0.05)$ en todo el ciclo de cultivo. El incremento en la densidad de población de 6.25 a 9.25 plantas $\mathrm{m}^{-2}$ causó un aumento de la RI en $11 \%(\mathrm{P} \leq 0.05)$ en el periodo EM-FF y $7 \%(\mathrm{P} \leq 0.01)$ durante todo el ciclo de cultivo. No hubo diferencias significativas ( $\mathrm{P}>$ 0.05) entre híbridos de maíz en cuanto a la RI en los tres periodos evaluados (Cuadro 2). La eficiencia en el uso de la radiación (EUR) sólo fue afectada por la fecha de siembra y a la densidad de población, pues la EUR aumentó en $30 \%(\mathrm{P} \leq 0.01)$ y $16 \%$ en el periodo EM-FF y durante todo el ciclo, respectivamente, en la fecha de siembra tardía (30/04/2008). De igual modo, al aumentar la densidad de población a 9.25 plantas por $\mathrm{m}^{2}$ incrementó la EUR en 17 \% para el periodo EM-FF y en $12 \%$ al considerar todo el ciclo de cultivo (Cuadro 2).

En maíz la RI varía en función del ambiente de (e.g., época del año, nubosidad) y de la capacidad que de las plantas para interceptar la radiación fotosintéticamente activa (RFA), así como de las prácticas de manejo y del genotipo (Otegui et al., 1995). Los tres factores de manejo agronómico evaluados afectaron en forma diferencial a la cantidad de RI en los periodos pre y post-floración, donde la dosis de $\mathrm{N}$ y la densidad de población fueron los únicos factores que modificaron la RI acumulada durante todo el ciclo de cultivo (Cuadro 2). Lo anterior es consistente con otros trabajos en el sentido de que al aumentar la disponibilidad de $\mathrm{N}$ y el número de plantas por unidad de superficie, por un lado se mejoran las condiciones de crecimiento y por otro el cultivo adquiere una mayor capacidad para interceptar RFA a través de una mayor cobertura por el dosel de las plantas (Carcova et al., 2003; D'Andrea et al., 2008). La EUR resultó afectada únicamente por la fecha de siembra y la densidad de población, y fue consistentemente mayor para el periodo pre-floración y menor para el periodo post-floración, lo cual concuerda con lo reportado por Cirilo y Andrade (1994) quienes señalaron que la EUR del maíz es mayor durante el periodo vegetativo y luego declina durante el llenado de grano y la madurez fisiológica, cuando las hojas senescen y reducen su tasa de fotosíntesis. 
Cuadro 1. Resumen mensual de temperaturas, radiación solar y precipitación durante el ciclo primavera verano 2008, en el Cerrillo Piedras Blancas, Toluca, México.

\begin{tabular}{lcccccc}
\hline & \multicolumn{3}{c}{ Temperatura $\left({ }^{\circ} \mathrm{C}\right)$} & & Radiación & \multicolumn{2}{c}{ Precipitación } \\
\cline { 2 - 3 } \cline { 5 - 6 } Mes & Máxima & Mínima & Media & & $\left(\mathrm{MJ} \mathrm{m}^{-2} \mathrm{~d}^{-1}\right)$ & $(\mathrm{mm})$ \\
\hline Abril & 26.8 & 5.3 & 14.2 & & 21 & 164 \\
Mayo & 25.9 & 6.4 & 14.6 & & 22 & 160 \\
Junio & 25.4 & 5.7 & 13.3 & & 19 & 213 \\
Julio & 24.2 & 9.6 & 14.2 & & 19 & 235 \\
Agosto & 25.0 & 8.4 & 13.9 & & 20 & 195 \\
Septiembre & 23.4 & 9.0 & 14.2 & & 16 & 128 \\
Octubre & 23.8 & 3.8 & 12.2 & & 18 & 27 \\
Noviembre & 24.0 & -1.3 & 9.9 & 13.3 & 19 & 0 \\
Promedio & 24.8 & 5.9 & & 19 & Total: 1122
\end{tabular}

Fuente: Estación climática de la Facultad de Ciencias Agrícolas de la Universidad Autónoma del Estado de México.

Cuadro 2. Medias de radiación interceptada acumulada y de eficiencia en el uso de la radiación, durante los periodos: emergencia-floración femenina (EM-FF), floración femenina-madurez fisiológica (FF-MF) y emergencia-madurez fisiológica (EM-MF), en dos fechas de siembra, dos dosis de nitrógeno, dos densidades de población y tres híbridos de maíz. Toluca, México.

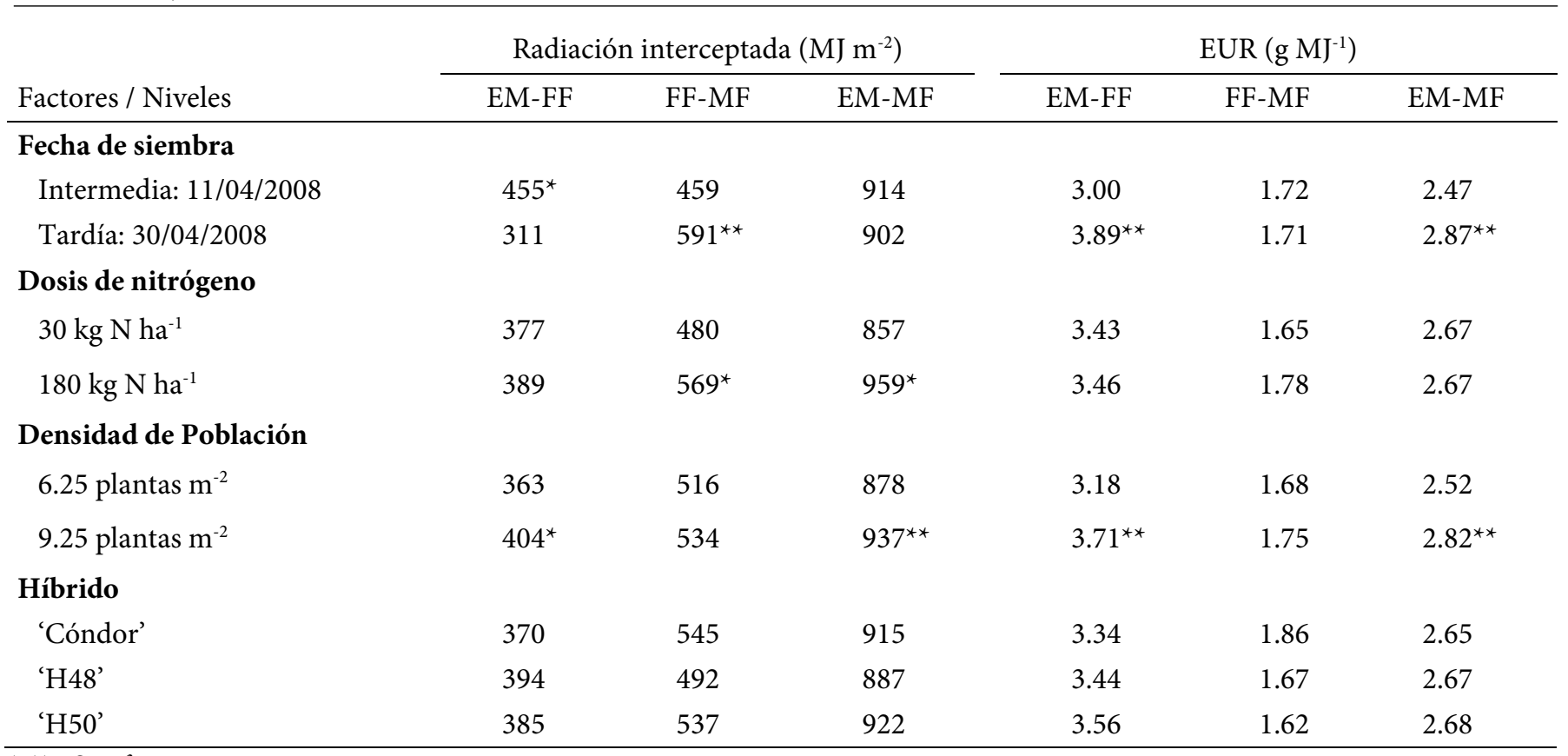




\section{Rendimiento de grano y sus componentes}

En la fecha de siembra tardía el rendimiento de grano por parcela $(\mathrm{RG})$ y el número de granos por $\mathrm{m}^{2}(\mathrm{NG})$ disminuyeron significativamente $(\mathrm{P} \leq 0.05)$ en 20 y $22 \%$ respectivamente. El incremento en la dosis de $\mathrm{N}$ de 30 a $180 \mathrm{~kg} \mathrm{ha}^{-1}$ aumentó $(\mathrm{P} \leq 0.01)$ el RG en $43 \%$ como consecuencia de incrementos en sus principales componentes: NG (28 \%) y PMG (12.3\%), así como en el peso de grano por planta (PGP) y número de granos por planta (NGP) cuyos incrementos fueron de 25 y $11 \%$, respectivamente (Cuadro 3). Al aumentar la densidad de población de 6.25 a 9.25 plantas $\mathrm{m}^{-2}$, PGP, NGP y PMG disminuyeron $(\mathrm{P} \leq 0.05)$; sin embargo, el RG aumentó $(\mathrm{P} \leq 0.05)$ debido a que el $\mathrm{NG}$ incrementó en $17 \%$ $(\mathrm{P} \leq 0.01)$. RG y NG difirieron entre híbridos $(\mathrm{P} \leq 0.05)$, 'Cóndor' y 'H-50' alcanzarón RG de 7 t ha ${ }^{-1}$ (698 y 693 g $\left.\mathrm{m}^{-2}\right)$ y 'Condor' destacó además por su mayor NG (2033 granos por $\mathrm{m}^{2}$ ) (Cuadro 3 ).

En los tres híbridos se observó que la RI acumulada durante todo el ciclo de cultivo y el NG se relacionaron positiva y significativamente $(\mathrm{P} \leq 0.05)$; el mayor $\mathrm{NG}$ se obtuvo con la dosis más alta de $\mathrm{N}$ en los híbridos ' $\mathrm{H}-48$ ' y 'H-50' (Figuras 1A, B y C). Durante el periodo crítico
(PC) de $15 \mathrm{~d}$ pre a $15 \mathrm{~d}$ post-floración, el NG se incrementó linealmente hasta que la RI acumulada durante este periodo alcanzó un umbral entre 266 a 269 MJ $\mathrm{m}^{-2}$, a partir del cual el NG incrementó independientemente de la cantidad de RI, debido principalmente al efecto de la mayor dosis de $\mathrm{N}$ en el suelo (Figuras 1D, E, F). Resultados similares fueron reportados en otras investigaciones (Otegui et al., 1995; Andrade et al., 1999), en donde el mejor ambiente de crecimiento se tuvo con la mayor disponibilidad de $\mathrm{N}$ cuando el incremento en el número de granos (NG) es independiente de la RI que rebase un umbral determinado. Por otro lado, cuando la fertilización nitrogenada es la fuente de variación del rendimiento, éste incrementa tanto por aumentos en el NG como en el PG (Melchori y Caviglia, 2008). En este trabajo, los rendimientos obtenidos con la dosis baja de nitrógeno parecen altos porque había una alta cantidad $\mathrm{N}$ disponible al momento de la siembra $\left(162 \mathrm{~kg} \mathrm{ha}^{-1}\right)$, puesto que en el ciclo anterior al experimento el terreno no fue cultivado. No obstante, las diferencias entre los dos niveles de fertilización nitrogenada fueron significativas $(\mathrm{P} \leq 0.05)$.

Cuadro 3. Medias de rendimiento de grano por parcela (RG), peso de grano por planta (PGP), número de granos por planta (NGP), peso de mil granos (PMG) y número de granos por $\mathrm{m}^{2}$ (NG) en dos fechas de siembra, dos dosis de nitrógeno, dos densidades de población y tres híbridos de maíz. Toluca, México.

\begin{tabular}{|c|c|c|c|c|c|}
\hline Factores/ Niveles & $\begin{array}{c}\mathrm{RG} \\
\left(\mathrm{g} \mathrm{m}^{-2}\right) \\
\end{array}$ & $\begin{array}{c}\text { PGP } \\
\text { (g/planta) }\end{array}$ & NGP & $\begin{array}{c}\text { PMG } \\
\text { (mg/grano) }\end{array}$ & $\begin{array}{c}\mathrm{NG} \\
\left(\text { granos } \mathrm{m}^{-2}\right)\end{array}$ \\
\hline \multicolumn{6}{|l|}{ Fecha de siembra } \\
\hline Intermedia: $11 / 04 / 08$ & $750^{*}$ & 111 & 316 & 348 & $2150^{*}$ \\
\hline Tardía: 30/04/2008 & 600 & 105 & 291 & 360 & 1670 \\
\hline \multicolumn{6}{|l|}{ Dosis de nitrógeno } \\
\hline $30 \mathrm{~kg} \mathrm{~N} \mathrm{ha}^{-1}$ & 555 & 96 & 287 & 334 & 1676 \\
\hline $180 \mathrm{~kg} \mathrm{~N} \mathrm{ha}^{-1}$ & $794^{* *}$ & $120^{*}$ & 320 & $375^{\star \star}$ & $2143^{* *}$ \\
\hline \multicolumn{6}{|l|}{ Densidad de Población } \\
\hline 6.25 plantas $\mathrm{m}^{-2}$ & 645 & $118^{* *}$ & $324^{*}$ & $365^{\star \star}$ & 1761 \\
\hline 9.25 plantas $\mathrm{m}^{-2}$ & $705^{\star}$ & 97 & 283 & 343 & $2059^{* *}$ \\
\hline \multicolumn{6}{|l|}{ Híbrido } \\
\hline 'Cóndor' & $698^{*}$ & 112 & $324^{*}$ & 345 & $2033^{\star}$ \\
\hline 'H48’ & 632 & 110 & 282 & 352 & 1798 \\
\hline 'H50’ & 693 & 111 & 304 & 365 & 1899 \\
\hline
\end{tabular}

${ }^{\star},{ }^{* *}=$ Significativo a 0.05 y 0.01 , respectivamente. 

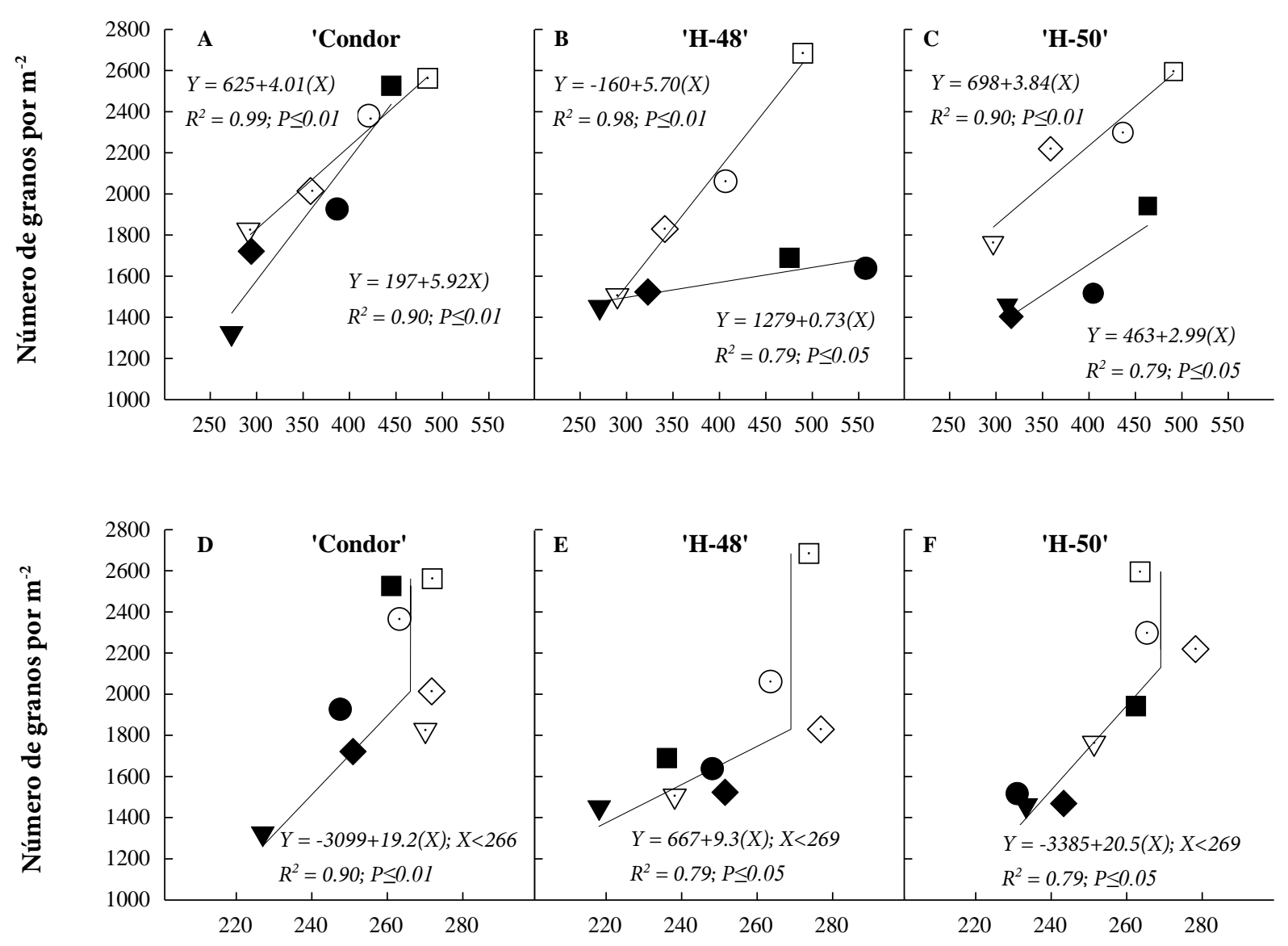

Radiación interceptada acumulada durante el periodo crítico $\left(\mathrm{MJ} \mathrm{m}^{-2}\right)$

\begin{tabular}{|c|c|c|c|}
\hline \multicolumn{2}{|c|}{$30 \mathrm{~kg} \mathrm{~N}$ ha $^{-1}$} & \multicolumn{2}{|c|}{$180 \mathrm{~kg} \mathrm{~N} \mathrm{ha}^{-1}$} \\
\hline FS:11/04/08; $6.25 \mathrm{pl} \mathrm{m}^{-2}$ & FS:11/04/08; $9.25 \mathrm{pl} \mathrm{m}^{-2} \odot$ & FS:11/04/08; $6.25 \mathrm{pl} \mathrm{m}^{-2} \square$ & FS:11/04/08; $9.25 \mathrm{pl} \mathrm{m}^{-2}$ \\
\hline FS:30/04/08; $6.25 \mathrm{pl} \mathrm{m}^{-2}$ & FS:30/04/08; $9.25 \mathrm{pl} \mathrm{m}^{-2} \nabla$ & FS:30/04/08; $6.25 \mathrm{pl} \mathrm{m}^{-2} \diamond$ & FS:30/04/08; $9.25 \mathrm{pl} \mathrm{m}^{-2}$ \\
\hline
\end{tabular}

Figura 1. Número de granos por metro cuadrado en función a la radiación interceptada acumulada durante el periodo emergencia-madurez fisiológica (A, B, C) y durante el período crítico (C, D, E) en tres híbridos de maíz ('Cóndor', 'H-48' y ' $\mathrm{H}$-50') evaluados en dos fechas de siembra (11/04/08 y 30/04/08), dos dosis de nitrógeno (30 y $\left.180 \mathrm{~kg} \mathrm{~N} \mathrm{ha}^{-1}\right)$ y dos densidades de población $\left(6.25\right.$ y 9.25 plantas $\left.\mathrm{m}^{-2}\right)$. Toluca México.

La fecha de siembra, dosis de $\mathrm{N}$ y densidad de población causaron una variación amplia en el RG y en sus principales componentes. Los tres híbridos sembrados en la fecha intermedia (11/04/2012) con dosis de nitrógeno alta $\left(180 \mathrm{~kg} \mathrm{~N} \mathrm{ha}^{-1}\right)$ y densidad de población alta $\left(9.25\right.$ plantas por $\mathrm{m}^{2}$ ) expresaron el mayor RG, debido a su mayor NG y alto PG (Figura 2). En los tres híbridos, el NG se relacionó positivamente $(\mathrm{P} \leq 0.05)$ con el RG, y el NG aumentó significativamente cuando se incrementó la dosis de $\mathrm{N}$ dentro de una misma fecha de siembra y densidad de población (Figuras 2A, 2B y 2C). El PG no mostró una relación consistente con el RG (Figuras 2D, 2E, 2F). Sólo en el híbrido 'Cóndor' crecido con la dosis de nitrógeno más baja $\left(30 \mathrm{~kg} \mathrm{~N} \mathrm{ha}^{-1}\right)$ se encontró una relación lineal negativa $(\mathrm{P} \leq 0.01)$ entre $\mathrm{PG}$ y RG (Figura 3D), lo cual se atribuye a un efecto compensatorio, ya que los tratamientos que obtuvieron mayor NG en este genotipo y esta dosis de N (Figura 2A), mostraron menor PG (Figura 2D). 
NG es el principal componente del rendimiento de grano en maíz que es muy sensible a los cambios ambientales en radiación y temperatura, y a prácticas agronómicas como riego y fertilización. En contraste, el peso final de grano (PG) es más estable y depende de la tasa de crecimiento en el periodo cercano a prefloración, y de la duración y tasa de llenado de grano en el periodo postfloración (Andrade et al., 1999; Borrás y Otegui, 2001; Lee y Tollenaar, 2007). En este trabajo el PG también fue sensible al manejo agronómico, ya que aumentó $(\mathrm{P} \leq 0.05)$ cuando la dosis de $\mathrm{N}$ aumentó de 30 a $180 \mathrm{~kg} \mathrm{ha}^{-1}$ (Figuras 2D, 2E, 2F).

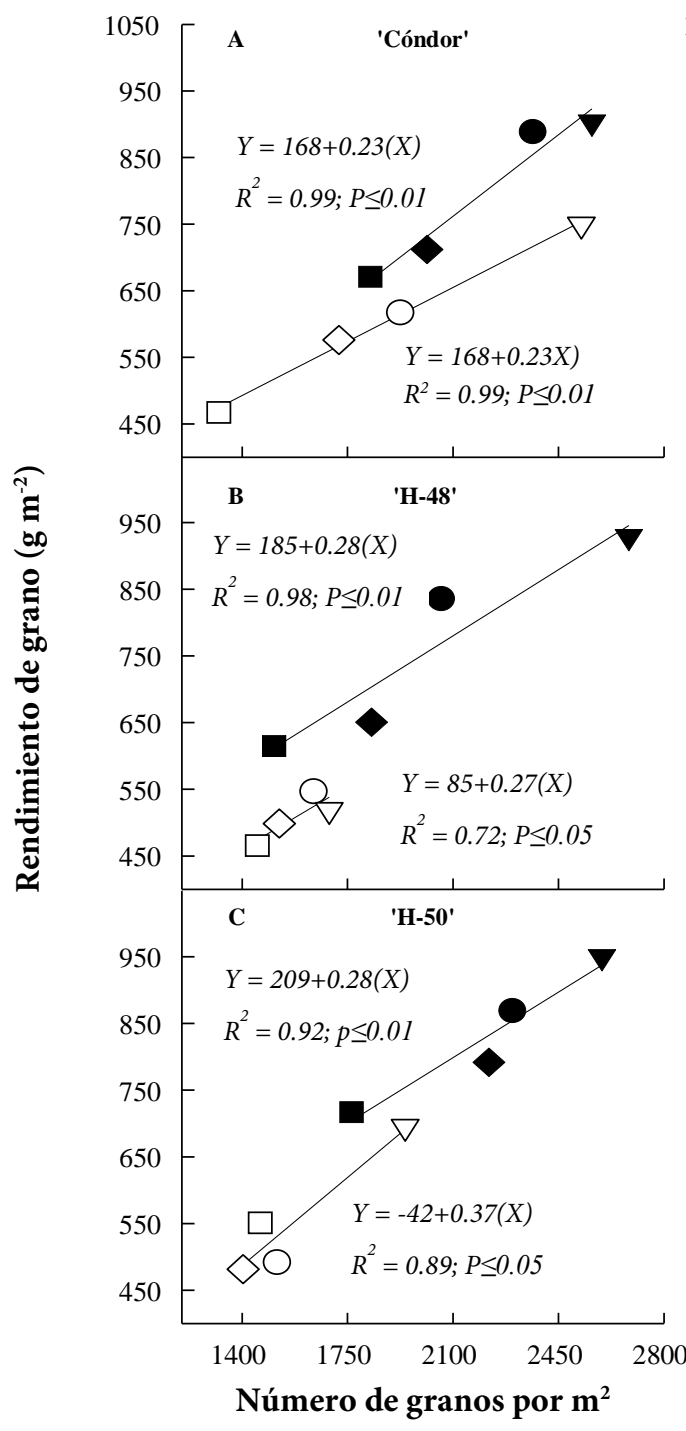

$30 \mathrm{~kg} \mathrm{~N}$ ha

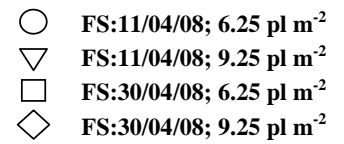

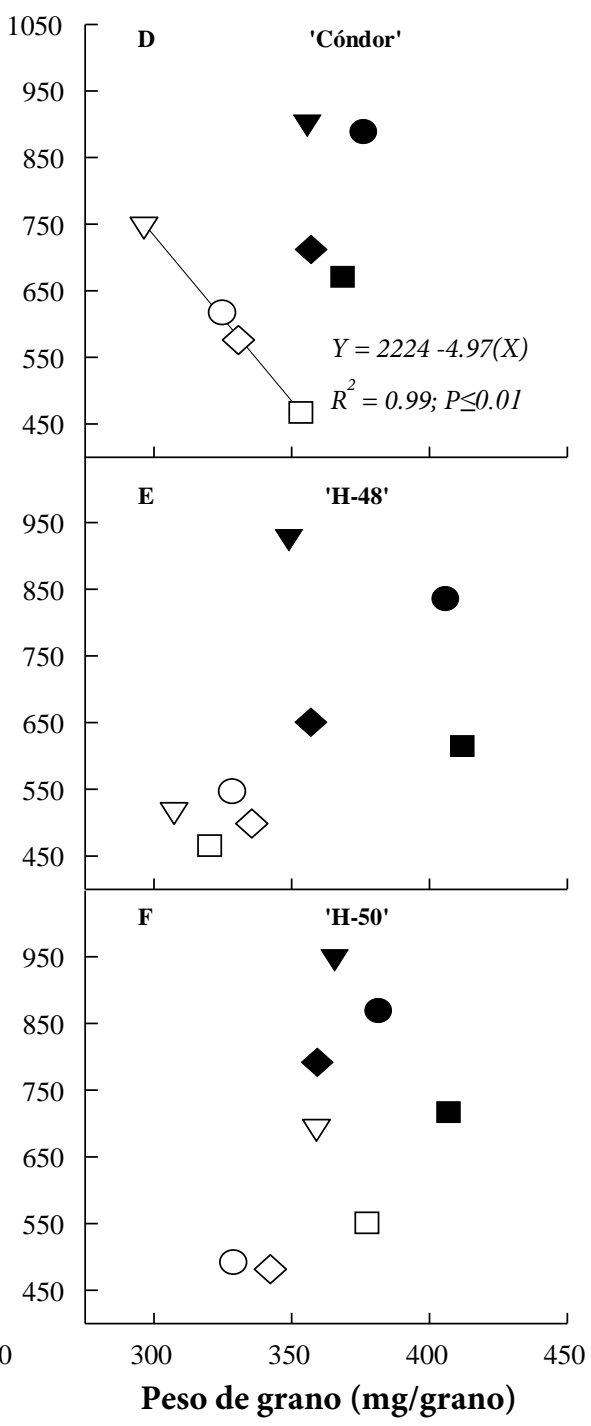

$180 \mathrm{~kg} \mathrm{~N}$ ha

FS:11/04/08; $6.25 \mathrm{pl} \mathrm{m}^{-2}$

FS:11/04/08; $9.25 \mathrm{pl} \mathrm{m}^{-2}$

FS:30/04/08; $6.25 \mathrm{pl} \mathrm{m}^{-2}$

FS:30/04/08; $9.25 \mathrm{pl} \mathrm{m}^{-2}$

Figura 2. Relaciones entre el rendimiento de grano y el número de granos por $\mathrm{m}^{2}(\mathrm{~A}, \mathrm{~B}, \mathrm{C})$ y entre el rendimiento de grano y peso de grano (D, E, F) en tres híbridos de maíz ('Cóndor', 'H-48' y 'H-50') evaluados en dos fechas de siembra (11/04/08; 30/04/08), dos dosis de nitrógeno y dos densidades de población (6.25 y 9.25 plantas $\left.\mathrm{m}^{-2}\right)$. Toluca, México. 


\section{CONCLUSIONES}

Las prácticas de manejo agronómico y el potencial genético de los híbridos influyeron significativamente sobre la eficiencia en el uso de la radiación y en el rendimiento de grano del maíz en Valles Altos de México. El incremento en la dosis de $\mathrm{N}$ y de la densidad de población aumentó la radiación interceptada a nivel de cultivo, y en consecuencia mejoraron las condiciones de crecimiento para la fijación de un mayor número de granos por unidad de superficie. El rendimiento de grano de los tres híbridos estudiados pudo ser explicado por el número de granos por unidad de superficie, más que por el peso individual de los granos. Destacó el híbrido 'Cóndor' por su capacidad para producir un mayor número de granos por unidad de superficie.

\section{AGRADECIMIENTOS}

Al Consejo Nacional de Ciencia y Tecnología (CONACYT) por el apoyo otorgado a través de la beca para la realización de los estudios de maestría en ciencias de la primera autora. Este trabajo formó parte del Proyecto de Investigación: Atributos Ecofisiológicos en Híbridos Comerciales de Maíz de Valles Altos, financiado por la Universidad Autónoma del Estado de México (Clave 2721/2008U).

\section{BIBLIOGRAFÍA}

Andrade F H, M A Ferreiro (1996) Reproductive growth of maize, sunflower and soybean at different source levels during grain filling. Field Crops Res. 48:155-165.

Andrade F H, C Vega, S A Uhart, A Cirilo, M Cantarero, O Valentinuz (1999) Kernel number determination in maize. Crop Sci. 39:453-459.

Andrade F H, V O Sadras, C R C Vega, L Echarte (2005) Physiological determinants of crop growth and yield in maize, sunflower and soybean: Their application to crop management, modeling and breeding. J. Crop Improv. 14:51-101.

Birch C J, G L Hammer, K G Rickert (1999) Dry matter accumulation and distribution in five cultivars of maize (Zea mays): relationships and procedures for use in crop modeling. Aus. J. Agric. Res. 50:513-27.

Borrás L, M E Otegui (2001) Maize kernel weight response to postflowering source-sink ratio. Crop Sci. 49:186-1822.

Cárcova J, L Borrás, M E Otegui (2003) Ciclo ontogénico, dinámica del desarrollo y generación del rendimiento y la calidad en maíz. In: Producción de Granos. Bases Funcionales para su Manejo; Ed. Facultad de Agronomía, Universidad de Buenos Aires. Buenos Aires, Argentina, pp:132-157.

Cirilo A G, F H Andrade (1994) Sowing date and maize productivity: I. Crow growth and dry matter partitioning. Crop Sci. 34:10391043.

D'Andrea K E, M E Otegui, A G Cirilo (2008) Kernel number determination differs among maize hybrids in response to nitrogen. Field Crops Res. 105:228-239.

Edwards J T, L C Purcell, E D Vories (2005) Light interception and yield potential of short-season maize (Zea mays L.) hybrids in the midsouth. Agron. J. 97:225-234.

García E (1988) Modificaciones al Sistema de Clasificación Climática de Köppen. Instituto Nacional de Geografía. Universidad Nacional Autónoma de México. $246 \mathrm{p}$

Gallo W P, C S T Daughtry (1986) Techniques for measuring intercepted and absorbed photosynthetically active radiation in crop canopies. Agron. J. 78:752-756.

Jandel Scientific (1992) Table Curve: Curve fitting software. Jandel Scientific., Corte madera, C. A.

Lee E A, M Tollenaar (2007) Physiological basis of successful breeding strategies for maize grain yield. Crop Sci. 47 (Suppl. 3): S202S215.

Melchori R J M, O P Caviglia (2008) Maize kernel growth and kernel water relations as affected by nitrogen supply. Field Crops Res. 108:198-205.

Monteith J L (1977) Climate and the efficiency of crop production in Britain. Philos. Trans. R. Soc. London, Serie B 281:277-294.

Otegui M E, M G Nicolini, R A Ruiz, P Dodds (1995) Sowing date effects on grain yield components for different maize genotypes. Agron. J. 87:29-33.

Ritchie S W, J J Hanway (1982) How a corn plant crop develops. Special Report 48. Iowa State University of Science and Technology. Cooperative Extension Service, Ames, IA.

SAS Institute (1999) SAS system version 8.0. SAS Institute, Cary, NC.

Sinclair T R, R C Muchow (1999) Radiation use efficiency Adv. Agron. 65:215-265.

Tollenaar M, A Aguilera (1992) Radiation use efficiency of old and a new maize hybrids. Agron. J. 84:536-541.

Tollenaar M, E A Lee (2002) Yield potential, yield stability and stress tolerance in maize. Field Crops Res. 75:161-169. 